\title{
A Clinical Review of First Seizures in Adult Patients
}

\section{Jose de Jesus Vidall Mayo*}

Department of Internal Medicine, National Institute of Medical Sciences and Nutrition, Universidad Nacional Autónoma de México (UNAM) Mexico

${ }^{*}$ Corresponding author: Jose de Jesus Vidal Mayo, Critical care fellowship, Department of Internal Medicine, National Institute of Medical Sciences and Nutrition, Universidad Nacional Autónoma de México (UNAM), Mexico, E-mail: interstrok@hotmail.com

Received date: April 17, 2019; Accepted date: May 24, 2019; Published date: May 31, 2019

Citation: de Jesus Vidal Mayo J (2019) A Clinical Review of First Seizures in Adult Patients. Med Clin Rev Vol. 5 No. 2: 3.

Copyright: (C 2019 de Jesus Vidal Mayo J. This is an open-access article distributed under the terms of the Creative Commons Attribution License, which permits unrestricted use, distribution, and reproduction in any medium, provided the original author and source are credited.

\section{Abstract}

First seizures in adult patients are a common problem in clinical practice, and their management represents a challenge for physicians. The aim of this review is to develop a systematic clinical approach for the classification, diagnosis, and treatment of patients who have experienced a first seizure. This could help clinicians to recognize and treat this condition appropriately. Because these seizures can arise from several etiologies, it is essential to know if the event was a provoked (acute symptomatic) or unprovoked seizure. Thus, an adequate classification is important to establish the length of the diagnosis approach, the necessary treatment, and every individual prognosis. We found that patients diagnosed with a single unprovoked seizure require electroencephalography and magnetic resonance imaging. This will allow an assessment of the risk of recurrence and the identification of patients that follow the diagnosis criteria for epilepsy. We recommend individualized pharmacological therapy to reduce early seizure recurrence ( $\leq 2$ years) even if it can produce some adverse effects. Finally, we determine that there are still certain areas of uncertainty to promote future research in this topic.

Keywords: First seizure; Epilepsy; Recurrence; Treatment

\section{Introduction}

Seizures are a common problem in clinical practice being responsible for about $1 \%$ of hospital admissions and $3 \%$ of emergency room visits [1]. An epileptic seizure is a transient event of signs or symptoms due to abnormally excessive or synchronous neuronal activity in the brain [2]. In contrast, a convulsion is the motor manifestation of this abnormal neuronal activity [3]. A first seizure is defined as one or multiple seizures with recovery of awareness between them within a period of $\leq$ 24 hours [2].

Epilepsy-according the most recent definition of the International League Against Epilepsy (ILAE)-is a disease of the brain which also includes a single unprovoked seizure with a high recurrence risk over the next 10 years (at least 60\%) [3].
Therefore, it is essential to perform a correct classification of the patient's event because it establishes the management and prognosis. The aim of this review is to develop a systematic clinical approach for the classification, diagnosis, and treatment of these patients to help clinicians recognize and treat this condition appropriately.

\section{Epidemiology}

Approximately 1 of 10 people throughout their lives will present an isolated seizure [4]. The lifetime risk for epileptic seizures is between $8 \%$ to $10 \%$ and $2 \%-3 \%$ chance of developing epilepsy $[5,6]$. About $40 \%$ to $50 \%$ of the first seizures correspond to provoked (acute symptomatic) seizures $[7,8]$.

The incidence regarding first seizures in Europe is around 70 per 100,000 inhabitants per year and is twice as common in the worldwide poorest countries [9]. In the United States of America (USA) close to 150,000 people present a first unprovoked seizure annually [10]. Some studies have reported a bimodal pattern of presentation with a higher incidence for children $<1$ year of age (252.9 per 100,000 per year) and for adults $>75$ years (173.2 per 100,000 per year) [11]

There are no epidemiological data in the Mexican population regarding this entity. However, according to the epilepsy priority program there are about 2 million people with epilepsy in our country [12].

\section{Classification}

For this review, we classify the epileptic seizures in two large groups:

Acute symptomatic seizures (also called provoked seizures): They are in close temporal association with an acute damage to the central nervous system which may be of metabolic, toxic, structural, infectious, or inflammatory origin [13]. They are presumably the acute manifestation of the insult [14].

The temporal relationship in this definition is within the first week in stroke, head trauma, or anoxic encephalopathy. The active phase of the infections in the central nervous system (based on persistent clinical, laboratorial, or imaging findings) is not beyond 24 hours for severe metabolic derangement and 
between 7 to 48 hours since the last alcohol intake in alcohol withdrawal [13].

Unprovoked seizures (also called remote symptomatic seizures): They occur in the absence of a precipitating factor and may be conditioned by a static or progressive injury [14]. These ones are the kind of seizures closely related to epilepsy [15].

Seizures can also be classified according to their presumed clinical and electrographic patterns, as generalized (they arise and engage neural networks in both cerebral hemispheres), or focal (they engage neural networks only in one cerebral hemisphere) [16].

Other experts have suggested a different classification for this topic [17], but we prefer to divide first-time seizures in provoked and unprovoked seizures to avoid confusion with the nomenclature.

\section{Etiology}

Causes of acute symptomatic seizures can be categorized as follows: $[15,18,19]$

\section{Neurologic insults}

Traumatic Brain Injury (TBI), brain surgeries are included in this section. Some factors associated with seizure occurrence in this context are: age (more common in children), loss of awareness, amnesia for $>30$ minutes, and the presence of cerebral hemorrhages or subdural hematomas [20].

CNS infections: about $5 \%$ of the patients will have seizures during the acute phase [21]. The risk factors described are: encephalitis (14 times more frequently compared to meningitis), the etiological agent (most common in herpes simplex encephalitis-up to $40 \%$ to $60 \%$ of cases), age $>42$ years and $\leq 12$ in the Glasgow Coma Scale (GCS) score at admission [18,22].

Acute stroke: those patients with cerebral vein thrombosis present more frequently symptomatic seizures (up to $39 \%$ of cases) [23], followed by those ones with subarachnoid hemorrhage ( $6 \%$ to $18 \%$ of patients) [24], up to $16 \%$ of cases in intracerebral haemorrhage [25], and finally patients who have experienced ischemic stroke (4\% to 9\%) [26-28].

\section{Metabolic disorders}

Metabolic disorders are responsible for $2.9 \%$ to $5 \%$ of epileptic seizures in emergency departments [29,30]. In this group we can include sodium, calcium and magnesium derangements as well as glucose disorders, and the settings of acute liver failure, uremic syndrome, and thyroid emergencies.

If there are electrolytic disturbances, the probability for the development of seizures depends on the speed and severity of instauration $[18,19]$. In glucose disorders, seizures occur more frequently in the context of hyperglycemic crises (up to 25\%) particularly in the hyperglycemic hyperosmolar state, probably due to the antiepileptic effect of ketosis [19]- compared to hypoglycemia (7\% of cases of severe hypoglycemia) [31]. The associated alterations and their respective cut-off points related to seizures are represented in the Table $1[13,15,19]$.

Table 1 Metabolic disorders and their respective cut-off related to seizures.

\begin{tabular}{|l|l|}
\hline Parameter & Cut-off points \\
\hline Sodium & Hyponatremia $<115 \mathrm{mmol} / \mathrm{L}$ \\
\hline Calcium & Hypocalcemia $<5 \mathrm{mg} / \mathrm{dL}$ \\
\hline Magnesium & Hypomagnesemia $<0.8 \mathrm{mg} / \mathrm{dL}$ \\
\hline \multirow{2}{*}{ Glucose } & Hypoglycemia $<36 \mathrm{mg} / \mathrm{dL}$ \\
\cline { 2 - 2 } & Hyperglycemia $\geq 400-450 \mathrm{mg} / \mathrm{dL}$ \\
\hline
\end{tabular}

\section{Medications}

Around $6 \%$ of first-time seizures are produced by pharmacological toxicity [32]. There are more than 250 drugs related to seizures with an adverse effect [33]. This association can occur either in their therapeutic use (very rare, only $0.08 \%$ [34]) and in overdosing [19]. Some drugs with an elevated risk for seizures are isoniazid, bupropion and venlafaxine [35]. Table 2 lists the most representative drugs [19,35-40].

Table 2 Common medications associated with acute symptomatic seizures.

\begin{tabular}{|c|c|}
\hline Drug class & Representative medications \\
\hline \multirow[t]{4}{*}{ Antibiotics } & $\begin{array}{l}\text { Beta lactams: penicillins and } \\
\text { cephalosporins }\end{array}$ \\
\hline & $\begin{array}{l}\text { Carbapenems: imipenem-cilastatin, } \\
\text { meropenem and doripenem }\end{array}$ \\
\hline & Fluoroquinolones \\
\hline & Isoniazid \\
\hline Antivirals & Aciclovir \\
\hline Metilxantins & Theophyline \\
\hline \multirow[t]{4}{*}{ Antidepressants } & Tryciclic antidepressants \\
\hline & $\begin{array}{l}\text { Selective serotonin reuptake } \\
\text { inhibitors }\end{array}$ \\
\hline & Bupropion \\
\hline & Venlafaxin \\
\hline \multirow[t]{3}{*}{ Antipsychotics } & $\begin{array}{l}\text { First generation: chlorpromazine (the } \\
\text { highest risk), molindone, haloperidol, } \\
\text { fluphenazine, pimozide and } \\
\text { trifluoperazine. }\end{array}$ \\
\hline & Second generation: clozapine \\
\hline & Lithium \\
\hline Narcotics & $\begin{array}{l}\text { Meperidine, morphine and } \\
\text { propoxyphene }\end{array}$ \\
\hline Antiepileptic drugs & Carbamazepine, phenytoin \\
\hline Anticholinergic drugs & Dyphenhydramine \\
\hline Sodium channel blockers & Lidocaine \\
\hline
\end{tabular}




\section{Alcohol and toxics}

Alcohol abuse is associated from $20 \%$ to $40 \%$ of patients with seizures in emergency departments. Although alcohol abstinence is the most common setting, we must consider all the other possibilities that can produce first seizures in the patients [35].

Regarding substances of abuse, the most evident associations are abstinence from hypnotic-sedative drugs (benzodiazepines and barbiturates) and intoxication by sympathomimetics (cocaine and amphetamines) [18,35]. Other substances with uncommon association are heroin, mescaline, psilocybin, psilocin and synthetic ergotamine derivatives of diethylamide Dlysergic acid (LSD) [18].

\section{Others}

The seizures associated with Posterior Reversible Encephalopathy Syndrome (PRES) and eclampsia are considered within this group $[18,19]$.

About the etiology of unprovoked seizures, the most recent ILAE's classification suggests the following categories: genetic, structural, infectious, metabolic, immune and unknown (Table 3) [41].

Table 3 Main etiologies of unprovoked seizures and epilepsy.

\begin{tabular}{|c|l|}
\hline Etiology & Representative clinical conditions \\
\hline Structural & Stroke, TBI, tumors, infection. \\
\hline Genetic & Juvenil Myoclonic Epilepsy. \\
\hline Infectious & $\begin{array}{l}\text { Neurocysticercosis, tuberculosis, HIV, cerebral toxoplasmosis, } \\
\text { cerebral malaria, postinfectious epilepsy. }\end{array}$ \\
\hline Metabolic & Porphyria, aminoacidopathies or pyridoxine-dependent seizures. \\
\hline Immune & Autoimmune encephalitis. \\
\hline Unknown & Frontal lobe epilepsy. \\
\hline
\end{tabular}
[42].

It is the most common cause of epilepsy in Mexican adults

Some common etiologies should be considered according to the age group of the patient [18]:

15-34 years: traumatic brain injury, medications, alcoholtoxics and eclampsia.

35-64 years: brain tumors: primary-more frequent in lowgrade tumors-or metastatic tumors $[43,44]$

65 years: cerebrovascular diseases are the main etiology (overall $50 \%$ ) followed by traumatic brain injury and brain tumors $[38,45]$.

Some large series found as main causes of seizures in emergency departments the consumption of alcohol or toxics (19\%), TBI (7.8\%-16\%), cerebrovascular diseases (16\%) and infections $(15 \%)[29,46]$. The contribution of epilepsy in one of these studies was $6.8 \%$ and up to $41 \%$ of the seizures were classified as unknown [29]. In approximately $45 \%$ of patients with first-time seizures in emergency departments no cause is identified, and they are produced in $<10 \%$ by toxic-metabolic etiologies [47].

\section{Clinical Features}

Spectrum of clinical presentation depends of the underlying neuroanatomy, and can range from a focal motor crisis without altered awareness to generalized tonic-clonic seizures leading to a convulsive status epilepticus $[14,48]$.

The predominant one is as generalized seizures (86\%) [29]. Most of etiologies of acute symptomatic seizures (especially toxic-metabolic causes) produce generalized tonic-clonic seizures [16].

For epidemiological purposes, multiple seizures within 24 hours (seizure clusters) and first status epilepticus are considered as a first epileptic seizure since in themselves these events do not establish the diagnosis of epilepsy $[9,17,49]$. A study found no difference in the rate of recurrence between patients who experienced seizure clusters versus patients with a single seizure, regardless of etiology or treatment [50].

\section{Diagnostic Approach}

We suggest a systematic approach based on the following sequence [51,52]:

Define if the patient's event really corresponds to an epileptic seizure.

Establish the etiology and classify the event: provoked versus unprovoked seizure.

Identify if the event is about the first seizure or there have been previous events.

Complementary studies (laboratory, brain imaging, and electroencephalogram).

Categorize-if possible - the type of epileptic seizure, epilepsy, and/or epileptic syndrome.

Estimate the risk of seizure recurrence.

Define the need for treatment with Antiepileptic Drugs (AED).

\section{History and physical examination}

The initial clinical assessment should include a complete clinical history with emphasis on the family history as well as a physical examination directed towards the evaluation of vital signs, look for any neurological deficit, and the search of potential acute etiologies of the episode.

Diagnosis of epileptic seizures and epilepsy are eminently clinical, thus it is essential perform an adequate semiology of the event, so the description of the episode by the patient (if possible) or by a visual witness is of utmost relevance $[48,53]$. Also, it is recommended an early evaluation by the neurologist 
when this entity is suspected or if there is doubt about the diagnosis $[9,51,54]$.

The first step is to determine if we are facing a first epileptic seizure or corresponds to another spell, because an inadequate diagnosis is made between $16 \%-42 \%$ of the cases [55]. Syncope and Psychogenic Non-Epileptic Seizures (PNES) are the main differential diagnoses $[11,51,54,55]$. In the Table 4 are showed the main differential diagnoses of seizures in clinical practice $[47,54]$

Table 4 Most important seizures mimickers in clinical practice.

\begin{tabular}{|l|l|}
\hline $\begin{array}{l}\text { Non-neurologic differential } \\
\text { diagnosis }\end{array}$ & Neurologic differential diagnosis \\
\hline Syncope & \\
Delirium & Cerebrovascular disorder-Transient \\
Metabolic encephalopathies & Eschemic Attacks (TIAs) \\
& Episodic movement disorders \\
& Migraine \\
& Sleep disorders: cataplexy, \\
& parasomnia, REM behavior disorder, \\
& periodic limb movements during \\
& sleep. \\
& Psychogenic Non-Epileptic Seizures \\
& (PNES) \\
& Vestibulopathy \\
& Transient global amnesia \\
& Panic attacks \\
\hline
\end{tabular}

Some clinical features that suggest that the event is an epileptic seizure are postictal confusion, cyanosis, lateral tongue biting, preceding déjà $v u$ or jamais vu, confirmed unresponsiveness, head or gaze turning to one side, and the presence of limb rhythmic shaking or tonic posturing [9].

Syncope: It is a more frequent situation in the general population, especially the vasovagal. This diagnosis is supported by the presence of a situational factor and prodromal symptoms such as blurred vision, sweating, dizziness, nausea, dyspnea or palpitations and paleness. There may be associated myoclonic movements and even generalized tonic-clonic seizures during the episode due to cerebral hypoxia when the patients cannot adopt the supine position $[9,48]$

Psychogenic Non-Epileptic Seizures (PNES): These spells are characterized by behaviors closely resembling seizures with a psychiatric background, but they do not have the clinical and electrographic findings present during an epileptic seizure. They are frequent, accounting for up to $12 \%-18 \%$ of paroxysmal events with transient loss of awareness and 30\% to $50 \%$ of patients admitted to electroencephalographic monitoring units $[9,48]$. Some features that suggest this entity are prolonged duration of apparent loss of awareness with normal colour and/or oxygen saturation on room air, fluctuating motor activity, asynchronous movements with non-anatomical dissemination, side-to-side head or body movements, pelvic thrusting, ictal crying, presence of closed eyes during the event with resistance to its opening, and a rapid postictal recovery $[9,48,54]$.

About the second point of the approach sequence, we must rule out a provoked seizure in all patients who have experienced a first-time seizure, and only after that, we can label the seizure as unprovoked $[14,15]$.

Regarding the third point of the sequence, it is reported that between $7 \%$ to $50 \%$ of the patients who present for evaluation of a first seizure have experienced previous seizures, and that these ones are absences, focal or myoclonic seizures generally $[11,51]$. This fact is essential because it determines if the patient meets diagnostic criteria for epilepsy, which has a different treatment and prognosis than a single first seizure.

\section{General laboratory studies (blood cytometry, glucose, serum electrolytes)}

From $0 \%$ to $15 \%$ of the patients present alterations in these studies, but they have clinical significance rarely $(<5 \%)[55,56]$. Thus, they should be requested in an individualized way without use them routinely [5-57]. However, we suggest assessing serum glucose as well as serum electrolytes (sodium, calcium and magnesium) in every patient who has experienced a first seizure.

\section{Electroencephalography}

Electroencephalogram (EEG) must be performed in all patients who have experienced a first unprovoked seizure and shows significant abnormalities in $29 \%$ of these patients [57]. In addition, it helps to classify the seizure type (epileptic vs. nonepileptic, focal vs. generalized), to identify the seizure focus involved, and to characterize the seizure subtype. Therefore, EEG have implication to define the risk of recurrence and the treatment which could be employed in each patient $[48,55,57,58]$.

The sensitivity of a single routine record for epileptiform discharges is $\leq 50 \%$ in patients with epilepsy [48]. However, the diagnostic yield can increase by performing 3 or more serial records (up to $80 \%-90 \%$ ), performing records within first 24 hours after the epileptic seizure, and with stimulation maneuvers such as hyperventilation, photostimulation and sleep deprivation (up to $80 \%$ ) $[9,48,57]$. Some paroxysmal discharges with high epileptogenic potential $(>90 \%)$ are the anterior temporal lobe spikes, vertex spikes, generalized paroxysmal fast activity, generalized slow spike and wave and hypsarrhythmia [48]. Probability of seizure recurrence is estimated around $77 \%$ when there are epileptiform discharges [59].

Recent studies of patients who had experienced first-time unprovoked seizures in emergency departments found a higher diagnostic yield if the EEG was performed before their discharge ( $24 \%$ and $29.4 \%$ of them had abnormal records), which was relevant for starting AED treatment as well as the epilepsy diagnosis $[60,61]$. A study 24 hours videoelectroencephalography was performed within the first 7 days in patients who had first unprovoked seizures; epileptiform abnormalities were found in nearly $42 \%$ of them and were a risk factor for seizure recurrence (R.R. 2.25, C.I. 95\% 1.30-3.92) [62].

Among its main limitations are that it does not exclude the diagnosis of epilepsy, the lack of it availability in emergency 
departments, its cost and the misinterpretations of the records $[48,55]$.

\section{Brain imaging}

A neuroimaging study (CT or $\mathrm{MRI}$ ) must be performed in all patients with unprovoked first-time seizures [57].

Cranial Tomography (CT) scan shows significant alterations in approximately $10 \%$ of patients with this condition [57]. It is considered the first line imaging modality due to its wide availability in emergency departments and allows a faster diagnostic evaluation in patients who present an acute neurological deficit; especially, to exclude vascular etiologies or any situation that warrants neurosurgical treatment. Hence, CT scan must perform in every patient who has experienced a first seizure $[9,47,51,63]$.

Magnetic Resonance Imaging (MRI) is warranted in all patients with a first unprovoked seizure; unless, they have contraindications for its realization $[48,57]$.

$M R I$ is more sensitive than CT scan because allows a greater identification of significant lesions such as intraparenchymal hemorrhages, brain tumors, vascular malformations, posttraumatic changes, mesial temporal sclerosis, and malformations of cortical development [48].

Studies in this setting have reported that close to $30 \%$ of the patients was found a potentially epileptogenic alteration $[63,64]$, and of these patients about $12 \%$ had some specific pathological finding in presence of a normal CT [63]. The diagnostic yield of MRI is higher in patients with a first focal or provoked seizure (around 50\%), and performing an epilepsy protocol-specific brain MRI $[65,66]$.

\section{Lumbar puncture}

This procedure should be considered in patients with clinical suspicion of CNS infection, subarachnoid hemorrhage as well as in those with persistent impairment in mental status or are immunocompromised (even if they are afebrile) $[47,67]$

Some abnormalities in the cerebrospinal fluid of patients who experienced a first unprovoked seizure are hyperproteinorrachia (30\%) followed by pleocytosis (10\% of the cases, mean of 10.2 cells $/ \mathrm{mm}^{3}$ ). They are probably due to a transient disruption of the blood-brain barrier after the seizure [68]

\section{Other tests}

Toxicological profile, prolactin levels, electrocardiogram and pregnancy test.

Toxicological profile may be performed in selected cases where there is high clinical suspicion as in patients who have a toxindrome, known consumption of substances or altered mental status on examination. Nonetheless, there is not enough evidence to perform it routinely [56-58].

Prolactin levels have been measured above their normal value in patients who experienced an epileptic seizure and have been used to discriminate between seizures with altered awareness from Psychogenic Non-Epileptic Seizures (PNES). The above is applicable if there is a baseline determination of them ( 6 hours prior to the event), and these ones are compared against a new determination performed 10-20 minutes after the episode, which is complicated to implement in clinical practice. Besides, they cannot distinguish seizures from syncope because in both situations they can be elevated [69].

Other studies which should be performed in all patients in this setting are 12-lead electrocardiogram and pregnancy test in childbearing age women. The first one to look for any cardiac disturbance related to syncope, and the second one due to the association with eclampsia and pregnancy itself with epilepsy $[9,51]$

Depending on the context of each patient, it may be considered to request a test for detection of Human Immunodeficiency Virus (HIV), Holter study, echocardiogram, etc. $[51,70]$.

Summary of tests which we must perform in adult patients who have experienced a first seizure are shown in the Figure 1.

\begin{tabular}{l|}
\hline All patients (Emergency department assessment) \\
\hline Serum glucose \\
\hline Serum electrolytes: sodium, calcium and magnesium \\
\hline 12 leads electrocardiogram \\
\hline Neuroimaging study: CT scan \\
\hline Patients with a first unprovoked seizure \\
\hline Electroencephalogram \\
\hline Magnetic resonance imaging \\
\hline
\end{tabular}

Figure 1 Emergency tests to perform in all patients who have experienced a first seizure. 1 . It is preferred by a greater speed and availability in E.D. 2. Perform epilepsy-protocol specific MRI if it is available.

\section{Risk of Seizure Recurrence}

\section{Acute symptomatic seizures}

Patients with seizures resulting from acute brain insults (severe closed injury, acute hemorrhagic and ischemic stroke, brain surgery and CNS infections in active phase) have a lower recurrence rate (risk of seizure recurrence from $10 \%$ to $20 \%$ ) compared to those with remote symptomatic seizures $[14,17]$.

A study found that the risk of recurrence for a second seizure in patients who were diagnosed with stroke, TBI and CNS infections was significantly greater only for remote symptomatic seizures when these ones were compared against acute symptomatic seizures in the same setting; demonstrating that, in general, remote symptomatic seizures had a risk of recurrence greater than $60 \%$ [71].

\section{Unprovoked seizures}

Global risk of recurrence for a second seizure after a first unprovoked seizure is by $21 \%-45 \%$ within the first two years [72], and is particularly higher in the first 6 months (60\%-70\% of 
recurrences) $[51,73]$. The likelihood of recurrence after the second seizure increases to $57 \%$ at 1 year, and $73 \%$ at 4 years; besides, it is associated with a proportional increase in the frequency of episodes as well as a concomitant reduction in the free interval between subsequent seizures [74].

Patients at increased risk for seizure recurrence after a first unprovoked seizure according the American Academy of Neurology (AAN) Guideline Analysis are the following [72]:

Patients with prior brain lesion or insult (remote symptomatic). This group includes the seizures arising from stroke, traumatic brain injury, CNS infections, cerebral palsy and cognitive developmental disability (Level A of evidence).

EEG with epileptiform abnormalities (Level A of evidence)

Significant brain-imaging abnormality (Level B of evidence).

Nocturnal seizure (Level B of evidence).

Although there is no precise formula to estimate the risk of seizure recurrence in each patient, according to the model patients with history of an abnormal neurological status plus an abnormal electroencephalogram have a risk of recurrence greater than $60 \%$ at 3 and 5 years [75]. However, this tool have several limitations such as it does not consider the brain imaging, and it has not been validated in other studies $[17,72,75]$.

\section{Treatment with antiepileptic drugs}

The evidence regarding pharmacological treatment in this clinical entity and the ILAE's definition of epilepsy involves only unprovoked seizures. Thus, the treatment of provoked seizures corresponds to the specific of each underlying condition, and the use of antiepileptic drugs is to prevent further acute symptomatic seizures $[15,18]$.

Starting antiepileptic drug therapy in patients who have experienced a first unprovoked seizure reduces the risk for a second seizure by about 35\% within the subsequent 2 years [72]; however, it has no impact on long-term recurrence ( $\geq 3$ years after the first episode) and has not demonstrated any improvement in patients' quality of life $[72,76]$. In addition, pharmacological treatment can produce adverse effects (7\%-31\% of cases), although these ones are generally mild and reversible [72].

The evidence about efficacy of AED in the reduction of seizure recurrence arises from several randomized trials, but the main ones are: The First Seizure Trial Group study (FIR.ST) [77] and the European Multicenter Epilepsy and Single Seizure Study (MESS) [78]. In both trials it was found that the risk of seizure recurrence at 2 years was $50 \%$ less in the group of patients who had immediate AED therapy (adjusted relative risk $=0.5 ; \mathrm{Cl}$ $95 \%=0.3-0.6)$, but there was no impact on long-term remission rate of these patients $[52,77,78]$.

The only controlled, randomized and double blind trial from a study found a difference close to $52 \%$ between the group of patients who were treated with valproic acid after a first unprovoked seizure vs. the untreated group ( $4.3 \%$ in the treated group vs. $55.7 \%$ in the placebo group); nevertheless, it did not assess the clinical remission [79]. Others two smaller trials found differences of seizure recurrence by $34 \%$ and $49 \%$ between patients who were treated with AEDs and untreated patients after a single unprovoked seizure $[80,81]$. A meta-analysis of six studies in this setting showed an absolute risk reduction for seizure recurrence by $34 \%$ (CI 95\%=15\%-52\%) [56]

Some important factors to consider prior to begin AED therapy are the following $[14,15,17]$ :

Etiology and risk of seizure recurrence.

Seizure and epilepsy type.

Patient's age.

Patient's comorbidities: pregnancy, kidney or hepatic failure, etc.

Pharmacological properties of AED: pharmacokinetic and pharmacodynamic features, pharmacological interactions, individual tolerance, side effects, cost, and availability.

Inpatient vs. outpatient treatment. Some indications for hospital admission are shown in the Figure 2.

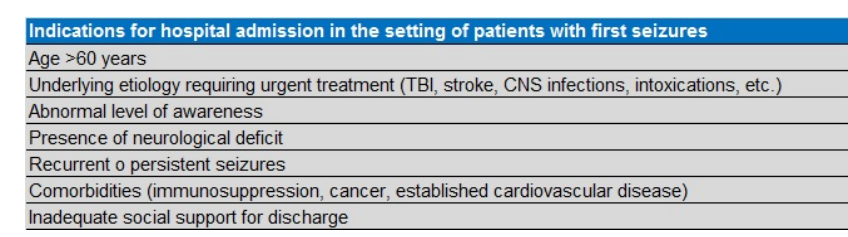

Figure 2 Indications for hospital admission.

Therefore, the decision about starting antiepileptic drug therapy must be individualized and should be always made in conjunction with patients $[72,82]$. We need to consider specific medical, social and employ features of each patient; although, the final decision corresponds to the specialist in this field (neurologist, epileptologist) $[9,17,55,58,71]$. In the Table 5 we provide a guide about the specific drugs to use according the seizure type in adults and in older patients ( $>60$ years) $[14,58,83]$.

Table 5 Preferred antiepileptic drugs according to the type of seizure.

\begin{tabular}{|l|l|}
\hline Seizure type & First line antiepileptic drugs \\
\hline Generalized tonic-clonic & Carbamazepine \\
& Lamotrigine \\
& Oxcarbazepine \\
& Sodium valproate \\
& Levetiracetam \\
& Topiramate \\
\hline \multirow{2}{*}{ Focal } & Carbamazepine \\
& Levetiracetam \\
& Oxcarbazepine \\
& Sodium valproate \\
& Phenytoin \\
& Zonisamide \\
\hline
\end{tabular}

This article is available from: http://medical-clinical-reviews.imedpub.com 


\begin{tabular}{|l|l|}
\hline Abscenses & $\begin{array}{l}\text { Euthosuximide } \\
\text { Sodium valproate } \\
\text { Lamotrigine }\end{array}$ \\
\hline Myoclonic & $\begin{array}{l}\text { Levetiracetam } \\
\text { Sodium valproate } \\
\text { Topiramate }\end{array}$ \\
\hline $\begin{array}{l}\text { Focal seizures in older } \\
\text { patients }\end{array}$ & $\begin{array}{l}\text { Lamotrigine } \\
\text { Gabapentine } \\
\text { Levetiracetam }\end{array}$ \\
\hline
\end{tabular}

\section{Length of therapy}

Around $70 \%$ of patients with new-onset epilepsy enter prolonged seizure remission during treatment with AED [84-86].

In patients with epilepsy, the factors associated with successful remission after starting AED therapy are a seizure free interval for more than 2 years and a normal neurological examination [87]. On the other hand, risk factors related to seizure recurrence after withdrawal are age $\geq 16$ years, need for more than one AED, seizures during AED therapy, history of generalized tonic-clonic or myoclonic seizures, and an abnormal EEG in prior year [88].

The largest trial about withdrawal of AEDs in patients with epilepsy who had been seizure-free for at least 2 years showed that $59 \%$ of them remained seizure-free at 2 years after weaning the medications. Also, it found that AED discontinuation doubles the risk of seizures for up to 2 years after stopping AED compared to continued treatment [89].

Consequently, it is recommended consider withdrawal AED therapy in those patients who have been seizure-free for at least 2 years and without high individual risk of seizure recurrence [90].

Patients with provoked seizures due to their low risk for developing epilepsy, the prophylaxis could be limited to 7 days (e.g. metabolic disorders), and from 1 to 6 months in patients with an acute brain insult [14].

\section{Follow Up and Prognosis}

It is suggested to perform a second evaluation by a neurologist within the first 4 weeks after the event in patients who experienced a first unprovoked seizure and those with suspected epilepsy, to complete the diagnostic tests (electroencephalogram and magnetic resonance imaging) as well as to define the need of AED therapy [91]

The use of specialized clinics in the evaluation of patients with first-time seizures allows a faster implementation of their study protocol and a faster diagnosis, with approximately $40 \%$ of patients evaluated in these centres meeting diagnostic criteria for epilepsy [92].

Some general recommendations for these patients are avoid the known precipitants of the seizures (if any) as well as the consumption of alcohol and other drugs which reduce the epileptic threshold, consider driving accompanied because them have increased risk of traffic accidents (up to $40 \%$ more), and avoiding high risk activities (water sports, heavy machinery use, etc.,) for at least 3-6 months with even larger periods for patients who have experienced unprovoked seizures [9,51,93].

Standardized Mortality Ratio (SMR) after a first unprovoked seizure is 2.3 , which is within the reported SMR by 2 to 4 for patients with epilepsy $[10,94,95]$. Mortality of symptomatic seizures is high, ranging from $10 \%$ to $40 \%$; however, it is difficult to distinguish between seizure-related deaths from deaths attributable to the underlying etiology [36].

\section{Discussion}

First seizures in adult patients are clinical significant events which require a systematic- multidisciplinary approach to identify those patients with a potential acute etiology, and separate them from those with unprovoked seizures and new onset epilepsy. The randomized trials and meta-analysis have found that the antiepileptic drugs do not reduce the risk for a second unprovoked seizure beyond the first two years since the first event; however, a seizure can be a terrifying event with medical and social consequences. Thus, the decision about starting pharmacological therapy should be individualized based in several factors mentioned previously.

This review summarizes the most recent scientific evidence in this field, and provides a practical framework for a suitable classification, diagnosis, and treatment of these patients as well as some elements to consider in the patient counselling after a first seizure.

There are several areas of uncertainty in this topic that are potential fields for future research. For example; some experts have questioned when a first unprovoked seizure is epilepsy; although, most of experts consider with the diagnosis of epilepsy those patients who have epileptiform abnormalities on EEG or a significant etiology in MRI. A prospective study by Lawn et al. reported that after the first seizure, a freedom seizure interval of 12 weeks reduced the initial risk of seizure recurrence of those patients (all of them had a basal risk of recurrence greater than $60 \%$ at 10 years); in other words, that the risk of seizure recurrence was time-dependent [96].

Also, the length of therapy in patients who have experienced a single seizure is uncertain because the available evidence concerning weaning AEDs arises from patients with epilepsy. Besides, it is necessary assess the effectiveness and the frequency of side effects of the new antiepileptic drugs because most of trials in this setting used older AEDs. Likewise, the effects at the epidemiological level regarding the diagnosis of epilepsy as well as the socioeconomic, legal and emotional consequences in patients receiving antiepileptic drug therapy are unknown $[55,71]$

Finally, it is important to evaluate the performance of the first seizure centres in other populations, and meet the challenges that they are facing in the management of these patients such as poor diagnostic accuracy of the referring doctors, underdetection of previous seizures, inadequate classification of seizures and low yield of investigations [97]. 


\section{Conclusions}

First seizures in adults are common in clinical practice. A systematic approach will help clinicians to classify the patients appropriately between those with provoked seizures and unprovoked seizures as well as those who meet diagnostic criteria for new-onset epilepsy, because the management and prognosis of each condition is very different. We must rule out a provoked seizure in all patients who have experienced a first seizure because they have a higher mortality and their treatment is defined by the underlying etiology. Patients who have experienced unprovoked seizures need to perform an EEG and a brain MRI to estimate the risk of seizure recurrence. Antiepileptic drug therapy must be individualized, without forgetting that only impacts in the early seizure recurrence (first 2 years) and may produce side effects. Every patient needs a tight follow-up, especially those who have experienced a first unprovoked seizure to complete their study protocol and to define the need of antiepileptic drug therapy.

\section{References}

1. Baroness Gould of Potternewton (2007) Wasted money, wasted lives: The human and economic cost of epilepsy in England. Report by All Party Parliamentary Group on Epilepsy.

2. Fisher RS, van Emde Boas W, Brume W, Elger C, Genton P, et al. (2005) Epileptic seizures and epilepsy: Definitions proposed by the International League Against Epilepsy (ILAE) and the international Burean for Epilepsy (IBE). Epilepsia 46: 470-472.

3. Fisher RS, Acevedo C, Arzimanoglou A, Bogacz A, Cross JH, et al. (2014) ILAE official report: A practical clinical definition of epilepsy. Epilepsia 55: 475-482.

4. Hauser WA, Annegers JF, Kurland LT (1993) Incidence of epilepsy and unprovoked seizures in Rochester, Minnesota: 1935-1984. Epilepsia 34: 453-468.

5. Hauser WA, Annegers JF, Rocca WA (1996) Descriptive epidemiology of epilepsy: Contributions of population-based studies from Rochester, Minnesota. Mayo Clin Proc 71: 576-586.

6. Berg AT, Shinar S (1991) The risk of seizure recurrence following a first unprovoked seizure. Neurology 41: 965-972.

7. Loiseau J, Loiseau P, Guyton M (1990) Survey of seizure disorders in the French Southwest: I. Incidence of epileptic syndromes. Epilepsia 31: 391-396.

8. Rizvi S, Ladino LD, Hernandez-Ronquillo L, Téllez-Zenteno JF (2017) Epidemiology of early stages of epilepsy: Risk of seizure recurrence after a first seizure. Seizure 49: 46-53.

9. Angus Leppan H (2014) First seizures in adults. BMJ 348: g2470.

10. Hauser WA, Beghi E (2008) First seizure definitions and worldwide incidence and mortality. Epilepsia 49: 8-12.

11. Martindale MD, Goldstein JN, Pallin DJ (2011) Emergency department seizure epidemiology. Emerg Med Clin N Am 29: 15-27.

12. Priority Epilepsy Program (2019) Programa prioritario de epilepsia, Registration Northeast Regional Meeting 2019. Mexico.

13. Beghi E, Carpio A, Forsgren L, Hesdorffer DC, Malmgren K (2010) Recommendation for a definition of acute symptomatic seizure. Epilepsia 51: 671-675.
14. Gavvala JR, Schueie SU (2016) New-onset seizure in adults and adolescents: A review. JAMA 316: 2657-2668

15. Espinosa-Jovel CA, Sobrino-Mejía FE (2014) Clinical approach to the first epileptic crisis in adults. Rev Neurol 58: 365-374.

16. Berg AT, Berkovic SF, Brodie MJ, Bucchalter J, Cross JH, et al. (2010) Revised terminology and concepts for organization of seizures and epilepsies: Report of the ILAE Commission on classification and terminology, 2005-2009. Epilepsia 51: 676-85.

17. Bergey GK (2016) Management of a first seizure. Continuum (Minneap Minn) 22: 38-50.

18. Beleza P (2012) Acute symptomatic seizures: A clinically oriented review. Neurologist 18: 109-119.

19. Karceski S (2014) Acute symptomatic seizures and systemic illnes. Continuum (Minneap Minn) 20: 614-623.

20. Burns J Jr, Hauser WA (2003) The epidemiology of traumatic brain injury: A review. Epilepsia 44: 2-10.

21. Ziai WC, Lewin JJ (2008) Update in the diagnosis and management of central nervous system infections. Neurol Clin 26: 47-68.

22. Kim MA, Park KM, Kim SE, Oh MK (2008) Acute symptomatic seizures in CNS infection. Eur J Neurol 15: 38-41.

23. Ferro JM, Canhao P, Bousser MG, Stam J, Barinagarrementeria F, et al. (2008) Early seizures in cerebral vein and dural sinus thrombosis: Risk factors and role of antiepileptics. Stroke 39: 1152.

24. Conolly ES, Rabinstein AA, Carhuapoma JR (2011) Guidelines for the management of aneurysmal subarachnoid hemorrhage: $A$ guideline for healthcare professionals from the American Heart Association/American Stroke Association. Stroke 43: 1711-1737.

25. Hemphill JC 3rd, Greenberg SM, Anderson CS, Becker K, Bendok $B R$, et al. (2015) Guidelines for the management of spontaneous intracerebral hemorrhage: A guideline for healhcare professionals from the American Heart Association/American Stroke Association. Stroke 46: 2032-2060.

26. Giroud M, Gras P, Fayolle H, André N, Soichot P, et al. (1994) Early seizures after acute stroke: A study of 1,640 cases. Epilepsia 35: 959.

27. Sung CY, Chu NS (1990) Epileptic seizures in thrombotic stroke. J Neurol 237: 166

28. Bladin CF, Alexandrov AV, Bellavance A, Bornstein N, Chambers B, et al. (2000) Seizures after stroke: A prospective multicenter study. Arch Neurol 57: 1617

29. Ong S, Talan DA, Moran GJ (2002) Neurocysticercosis in radiographically imaged seizure patients in U.S. emergency departments. Emerg Infect Dis 8: 608-613.

30. Hennemn PL, DeRoos F, Lewis RJ (1994) Determening the need for admission in patients with new onset seizures. Ann Emerg Med 24: 1108-1114.

31. Gutierrez A, Riggs JE (2002) Seizures and electrolyte imbalance. In Delanty, N. (ed.) Seizures: Medical causes and management. Totowa: Humana Press Inc. pp:85-106.

32. Chen HY, Albertson TE, Olson KR (2015) Treatment of druginduced seizures. Br J Clin Pharmacol 81: 412-419.

33. NA (2013). Physicians Desk Reference $2014,68^{\text {th }}$ ed. Montvale, N.J.: PDR Network. 
34. Garcia PA, Alldregue BK (2002) Medication-induced seizures. In Delanty N, (ed.) Seizures: Medical causes and management. Totowa: Humana Press Inc. pp:147-165.

35. Sharma AN, Hoffman RJ (2011) Toxin related seizures. Emerg Med Clin N Am 29: 125-139.

36. Sutter R, Ruegg S, Tschudin-Sutter S (2015) Seizures as adverse events of antibiotic drugs: A systematic review. Neurology 85:1332-1341.

37. Calandra G, Lydick E, Carrigan J (1988) Factors predisposin to seizures in seriously ill affected patients receiving antibiotics: Experience with imipenem/cilastatin. Am J Med 84: 911-918.

38. Martinez GJ, Hsia R (2011) Altered mental status from acyclovir. J Emerg Med 41: 55-58.

39. Montgomery SA (2005) Antidepressants and seizures: Emphasis on newer agents and clinical implications. Int J Clin Pract 59: $1435-1440$.

40. Davidson J (1989) Seizures and bupropion: A review. J Clin Psychiatry 50: 256-261.

41. Scheffer IE, Berkovic S, Capovilla G (2017) ILAE classification of the epilepsies: Position paper of the ILAE Commission for classification and terminology. Epilepsia 58: 512-521.

42. Medina MT, Rosas E, Rubio-Donnadieu F (1990) Neurocysticercosis as the main cause of the late-onset epilepsy in Mexico. Arch Intern Med 150: 325-327.

43. Van Breemen MS, Wilms EB, Vecht CJ (2007) Epilepsy in patients with brain tumours: epidemiology, mechanisms and management. Lancet Neurol 6: 421-430.

44. Ertuk Cetin O, Isler C, Uzan M, Ozkara C (2017) Epilepsy-related brain tumors. Seizure 44: 93-97.

45. Liu S, Yu W, Lu Y (2016) The causes of new onset epilepsy and seizures in the elderly. Neuropsychiatr Dis Treat 12: 1425-1434.

46. Annegers JF, Hauser WA, Lee JR (1995) Incidence of acute symptomatic seizures in Rochester, Minnesota, 1935-1984. Epilepsia 36: 327-33.

47. Jagoda A, Gupta K (2011) The emergency department evaluation of the adult patient who presents with a first-Time seizure. Emerg Med Clin N Am 29: 41-49.

48. St. Louis E, Cascino GD (2016) Diagnosis of epilepsy and related episodic disorders. Continuum (Minneap Minn) 22: 15-37.

49. NA (1993) Guidelines for epidemiologic studies on epilepsy. Commission on epidemiology and prognosis, International league against epilepsy. Epilepsia 34: 592-596.

50. Kho LK, Lawn ND, Dunne JW, Linto J (2006) First seizure presentation: Do multiple seizures on 24 hours predict recurrence? Neurology 67: 1047-1049.

51. Seneviratne $U$ (2009) Management of the first seizure: An evidence based approach. Postgrad Med J 85: 667-673.

52. Martínez-Juarez IE, Moreno J, Ladino LD, Castro N, HernandezVanegas L, et al. (2016) Diagnosis and treatment of non-triggered single epileptic seizures. Rev Neurol 63: 165-175.

53. Nowacki TA, Jirsch JD (2017) Evaluation of the first seizure patient: Key points in the history and physical examination. Seizure 49 : 54-63.

54. Cornes SB, Shih T (2011) Evaluation of the patient with spells. Continuum Lifelong Learning Neurol 17: 984-1009.
55. Jette N, Wiebe S (2016) Initial evaluation of the patient with suspected epilepsy. Neurol Clin 34: 339-350.

56. Wiebe S, Tellez-Zenteno JF, Shapiro M (2008) An evidence-based approach to the first seizure. Epilepsia 49: 50-57.

57. Krumholz A, Wiebe S, Gronseth G (2007) Practice paremeter: Evaluating an apparent unprovoked first seizure in adult (an evidence-based review): Report of the quality standards subcommittee of the American Academy of Neurology and the American Epilepsy Society. Neurology 69: 1996-2007.

58. Jackson MJ (2014) Concise guidance: Diagnosis and management of the epilipsies in adults (NICE). Clinical Medicine 17: 622-627.

59. Debicki DB (2017) Electroencephalography after a single unprovoked seizure. Seizure 49: 69-73.

60. Paliwal P, Wakerley BR, Yeo LL, Ali KM, Ibrahim I, et al. (2015) Early electroencephalography in patients with emergency room diagnoses of suspected new-onset seizures. Diagnostic yield and impact on clinical decision-making. Seizure 31: 22-26.

61. Wyman AJ, Mayes BN, Hernandez-Nino J, Rozario N, Beverly SK, et al. (2017) The first-time seizure emergency department electroencephalogram study. Ann Emerg Med 69: 184-191.

62. Chen T, Si Y, Chen D, Zhu L, Xu D, et al. (2016) The value of 24-hour video-EEG in evaluating recurrence risk following a first unprovoked seizure: A prospective study. Seizure 40: 46-51.

63. Ho K, Lawn N, Bynevelt M (2013) Neuroimaging of first-everseizure: Contribution of MRI if CT is normal. Neurol Clin Practice 3: 398-403.

64. Hakami T, Mcintosh A, Todaro M, Lui E, Yerra R, et al. (2013) MRIidentified pathology in adults with new-onset seizures. Neurology 81: 920-927.

65. Crocker CE (2016) Role of the neuroimaging in first seizure diagnosis. Seizure: Eur J Epilepsy 49: 74-78.

66. Crocker CE, Pohlmann-Eden B, Schmidt MH (2017) Role of neuroimaging in first seizure diagnosis. Seizure 49: 74-78.

67. Sampere AP, Villaverde FJ, Martínez-Menendez B (1992) First seizure in adults: A prospective study from the emergency department. Acta Neurol Scand 86: 134-138.

68. Zisimopoulou V (2016) Cerebrospinal fluid analysis after unprovoked first seizure. Functional Neurology 31: 191-197.

69. Chen DK, So YT, Fisher RS (2005) Use of serum prolactin in diagnosing epileptic seizures: Report of the therapeutics and technology assessment subcommittee of the American Academy of Neurology. Neurology 65: 668-675.

70. Krishnanmurthy KB (2016) In the clinic: Epilepsy. Ann Intern Med 164: 17-32.

71. Hesdorffer DC, Benn EK, Cascino GD, Hauser WA (2009) Is a first acute symptomatic seizure epilepsy? Mortality and risk for recurrent seizure. Epilepsia 50: 1102-1108.

72. Krumholz A, Wiebe S, Gronseth GS (2015) Evidence-based guideline: Management of an uprovoked first seizure in adults: Report of the guideline development subcommittee of the American academy of neurology and the American epilepsy society. Neurology 84: 1705-1713.

73. Hart YM, Sander JW, Johnson AL (1990) National general practice study of epilepsy recurrence after a first seizure. Lancet 336: 1271-1274. 
74. Hauser WA, Rich SS, Lee JR, Annegers JF, Anderson VE (1998) Risk of recurrent seizures affer two unprovoked seizures. N Engl J Med 338: $429-434$

75. Kim LG, Johnson TL, Marson AG, Chadwick DW (2006) Prediction of risk of seizure recurrence affer a single seizure and early epilepsy: further results from the MESS trial. Lancet Neurol 5: 317-322.

76. Davenport RJ (2015) Review: Immediate vs. deferred antiepileptics reduce recurrence at 1 to 2 years affer an unprovoked ffrst seizure. Ann Intern Med 163: JC8.

77. NA (1993) Randomized clinical trial on the efficacy of antiepileptic drugs in reducing the risk of relapse affer a ffrst unprovoked tonicclonic seizure. First seizure trial group (FIR.S.T. Group). Neurology 43: 478-483.

78. Marson A, Jacoby A, Johnson A (2005) Immediate versus deferred antiepileptic drug treatment for early epilepsy and single seizures: A randomized controlled trial. Lancet 365: 2007-2013.

79. Chandra B (1992) First seizure in adults: To treat or not to treat. Clin Neurol Neurosurg 94: 561-563.

80. Das CP, Sawhney IM, Lal V, Prabhakar S (2000) Risk of recurrence of seizures following single unprovoked idiopathic seizure. Neurol India 48: 357-360.

81. Gilad R, Lampl Y, Gabbay U, Eshel Y, Sarova-Pinhas I (1996) Early treatment of a single generalized tonic-clonic seizure to prevent recurrence. Arch Neurol 53: 1149-1152.

82. NA (2015) Diagnosis and management of epilepsy in adults. Scottish Intercollegiate Guidelines Network, Healthcare Improvement Scotland. Edinburgh.

83. Glauser T, Ben-Manachem B, Bourgeois B, Cnaan A, Guerreiro C, et al. (2013) Update ILAE evidence review of antiepileptic drug efficacy and effectiveness as initial monotherapy for epileptic seizures and syndromes. Epilepsia 54: 551-563.

84. Werhahn KJ, Trinka E, Dobesberger J (2015) A randomized, doubleblind comparison of antiepileptic drug treatment in the elderly with new-onset focal epilepsy. Epilepsia 56: 450-459.

85. Pohlmann-Eden B, Marson AG, Noack-Rink M (2016) Comparative effectiveness of levetiracetam, valproate and carbamazepine among elderly patients with newly diagnosed epilepsy. BMC Neurol 16: 149-153.

86. Schmidit D, Sillanpaa M (2017) Stoping epilepsy treatment in seizure remission: Good or bad or both? Seizure 44: 157-161.

87. NA (1996) Practice parameter: A guideline for discontinuing antiepileptic drugs in seizure-free patients. Report of the Quality Standards Subcommittee of the American Academy of Neurology. Neurology 47: 600-602.

88. NA (1993) Prognostic index for recurrence of seizures after remission of epilepsy. Medical research council antiepileptic drug withdrawal study group. BMJ 306: 1374-1378.

89. NA (1991) Randomised study of antiepileptic drug withdrawal in patient in remmision. Medical Research Council Antiepileptic Drug Withdrawal Study Group. Lancet 337: 1175-1180.

90. Braun KP, Schmidit D (2014) Stopping antiepileptic drugs in seizure-free patients. Curr Opin Neurol 27: 219-226.

91. NA (2012) The epilepsies: The diagnosis and management of the epilepsies in adults and children in primary and secondary care. Pharmacological update of clinical guideline 20. National Institute for Health and Clinical Excellence.

92. Rizvi S, Hernandez-Ronquillo L, Moien-Afshari F, (2016) Evaluating the single seizure clinic model. J Neurol Sci 367: 203-210.

93. Legg KT, Newton M (2017) Counselling adults who experience a first seizure. Seizure 49: 64-68.

94. Beghi $\mathrm{E}$, Leone $\mathrm{M}$, Solari A (2005) Mortality in patients with a first unprovoked seizure. Epilepsia 46: 40-42.

95. Sander JW (2013) Comorbidity and premature mortality in epilepsy. Lancet 382: 1618-1619.

96. Lawn N, Chan J, Lee J, Dunne J (2015) Is the first seizure epilepsyand when? Epilepsia 56: 1425-1431.

97. Jackson A, Teo L, Seneviratne U (2016) Challenges in the first seizure clinic for adult patients with epilepsy. Epileptic Disor 18: 305-314. 